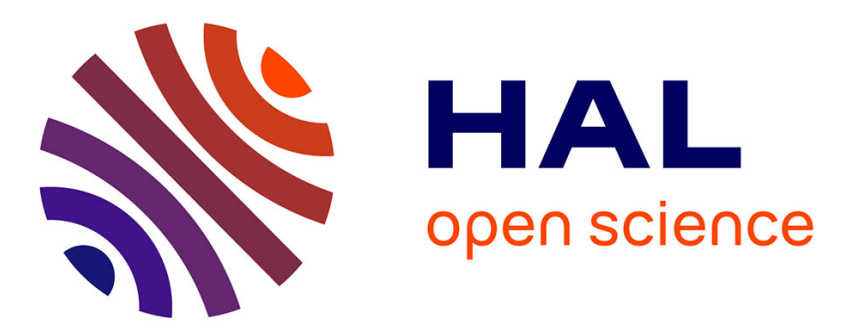

\title{
Precomputed Multiple Scattering for Light Simulation in Participating Medium
}

Beibei Wang, Nicolas Holzschuch

\section{To cite this version:}

Beibei Wang, Nicolas Holzschuch. Precomputed Multiple Scattering for Light Simulation in Participating Medium. Siggraph 2017 Talk, Jul 2017, Los Angeles, United States. pp.Article No. 35, 10.1145/3084363.3085037 . hal-01522404

\section{HAL Id: hal-01522404 \\ https://hal.inria.fr/hal-01522404}

Submitted on 15 May 2017

HAL is a multi-disciplinary open access archive for the deposit and dissemination of scientific research documents, whether they are published or not. The documents may come from teaching and research institutions in France or abroad, or from public or private research centers.
L'archive ouverte pluridisciplinaire HAL, est destinée au dépôt et à la diffusion de documents scientifiques de niveau recherche, publiés ou non, émanant des établissements d'enseignement et de recherche français ou étrangers, des laboratoires publics ou privés. 


\section{Precomputed Multiple Scattering for Light Simulation in Participating Medium}

\author{
Beibei Wang \\ Nanjing Univ. of Science \& Tech., Inria, Univ. \\ Grenoble-Alpes, CNRS, LJK
}

\author{
Nicolas Holzschuch \\ Inria, Univ. Grenoble-Alpes, CNRS, LJK
}

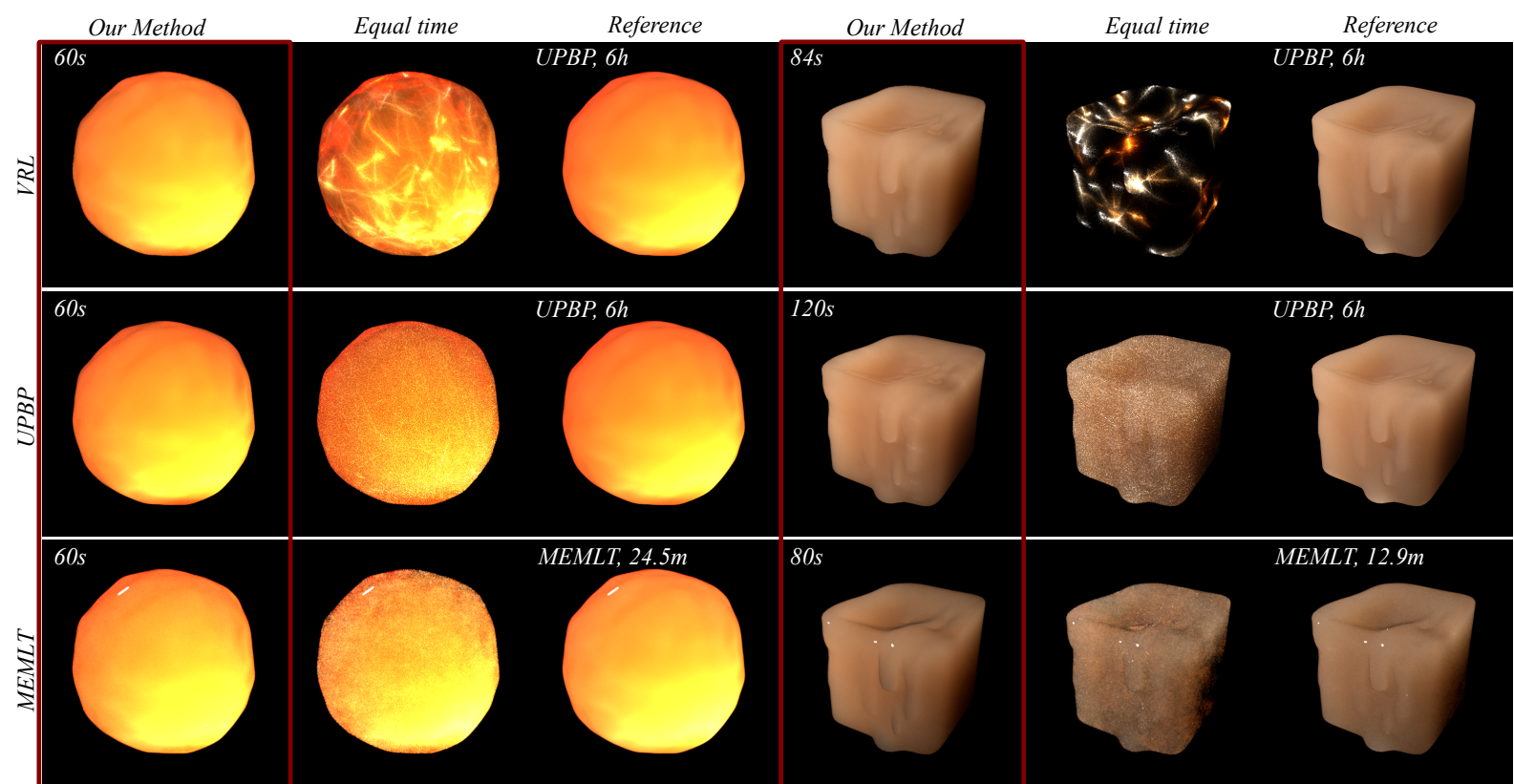

Figure 1: Our precomputed multiple scattering accelerates the convergence of existing algorithms for participating media.

\section{CCS CONCEPTS}

.Computing methodologies $\rightarrow$ Rendering;

\section{KEYWORDS}

Participating Media, Multiple Scattering, Precomputation

ACM Reference format:

Beibei Wang and Nicolas Holzschuch. 2017. Precomputed Multiple Scattering for Light Simulation in Participating Medium. In Proceedings of SIGGRAPH '17 Talks, Los Angeles, CA, USA, July 30 - August 03, 2017, 2 pages. DOI: http://dx.doi.org/10.1145/3084363.3085037

\section{OVERVIEW}

Illumination simulation involving participating media is computationally intensive. The overall aspect of the material depends on simulating a large number of scattering events inside the material. Combined, the contributions of these scattering events are a

Permission to make digital or hard copies of part or all of this work for personal or classroom use is granted without fee provided that copies are not made or distributed for profit or commercial advantage and that copies bear this notice and the full citation on the first page. Copyrights for third-party components of this work must be honored For all other uses, contact the owner/author(s).

SIGGRAPH '17 Talks, Los Angeles, CA, USA

(C) 2017 Copyright held by the owner/author(s). 978-1-4503-5008-2/17/07 ..\$15.00 DOI: http://dx.doi.org/10.1145/3084363.3085037 smooth illumination. Computing them using ray-tracing or photonmapping algorithms is expensive: convergence time is high, and pictures before convergence are low quality (see Figure 1). In this paper, we precompute the result of multiple scattering events, assuming an infinite medium, and store it in two $4 \mathrm{D}$ tables. These precomputed tables can be used with many rendering algorithms, such as Virtual Ray Lights (VRL), Unified Point Beams and Paths (UPBP) or Manifold Exploration Metropolis Light Transport (MEMLT), greatly reducing the convergence time. The original algorithm takes care of low order scattering (single and double scattering), while our precomputations are used for multiple scattering (more than two scattering events).

\section{PREVIOUS WORKS}

To address the specific issues caused by illumination simulation in participating media, Křivánek et al. [2014] extend the Photon Mapping method by combining beams, points and paths automatically in participating media, using multiple importance sampling (UPBP). Novák et al. [2012a; 2012b] used Virtual Ray Lights (VRL) or beams for light transport inside translucent materials. Jakob and Marschner [2012] extend Metropolis Light transport with a new mutation strategy based on manifold exploration, improving the sampling of specular and highly glossy paths. 

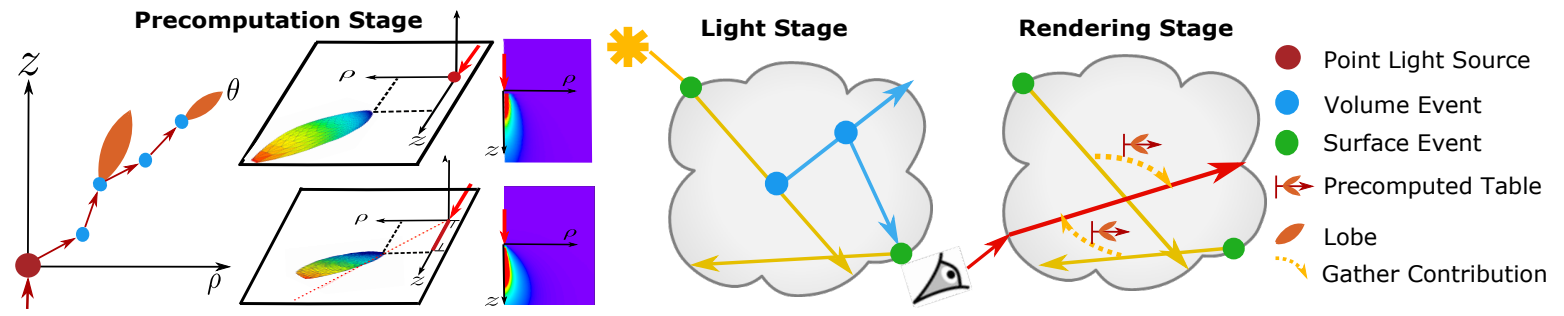

Figure 2: First, we precompute multiple scattering and store it in two tables, once per material. For rendering, we shoot and scatter rays, and then we store the rays (orange line) inside the medium after a surface event (green circle). We then use the precomputed tables to compute multiple scattering from these light rays (orange line) to the camera rays (red line).

Wang et al. [2016] presented a fast, point-based, method for global illumination with participating media, using a precomputed table for multiple scattering. Their method was limited to BiDirectional Path Tracing, making it harder to apply it to generic scenes. We extend their work on precomputation, adding multiple scattering from a segment instead of a point, and show this precomputation can be combined with many existing illumination simulation algorithms. Moon et al. [2007] also precomputed multiple scattering, but stored the result on a set of concentric spheres.

\section{PRECOMPUTED MULTIPLE SCATTERING}

In the precomputation step, we assume an infinite participating medium, with the same properties as the original medium. We run two types of precomputations: with a point directional light source and with a segment light source. In both cases, we shoot photons from the light source, let them travel in the medium, being scattered or absorbed, and accumulate their contributions in a table, exploiting the problem symmetry of revolution around the direction of propagation.

At each point, indexed by its cylindrical coordinates $(\rho, z)$ around this axis, we store the contributions as lobes in spherical coordinates (see Figure 2 and 3 ). We then compress these tables using a quadtree. Total computation time including compression is $\approx 1 \mathrm{mn}$ for highalbedo materials; storage cost after compression around $100 \mathrm{MB}$.

This storage method has higher resolution, both in space and angle, than the concentric spheres method [Moon et al. 2007] (see Figure 3).

\section{APPLICATION TO EXISTING ALGORITHMS}

We apply our precomputation method to several global illumination algorithms: VRL, UPBP, MEMLT. Each time, we let the original algorithm handle low-order scattering, and combine it with multiple scattering computed using our precomputed tables. .

For VRL, we shoot and scatter photons in the scene, but only store the first scattering event in the medium. We use these virtual rays as the source of multiple scattering, using our tables.

For UPBP, we store the first and the second scattering events in the medium (beams or points). We use these for single and double scattering. We use the single scattering events as the source for multiple scattering.

For MEMLT, for each generated seed path inside the medium, we modify it to a special path, replacing path vertices far from the surface medium by a special edge, representing multiple scattering, whose weight is computed using our tables. For mutation purposes, both end points of the special edge are considered as non-specular, and the end point from the light source can not be considered as the mutation source.

We have implemented our algorithm inside the Mitsuba Renderer [Jakob 2010] for VRL and MEMLT, and inside smallUPBP [Křivánek 2014] for UPBP.

Our algorithm increases convergence speed, at the expense of introducing bias. To reduce this bias, the user can decide to let the original algorithms handle more low-order scattering events (three or four scattering events, instead of two) and use our precomputed tables for larger order multiple scattering events.

\section{CONCLUSION AND FUTURE WORK}

We have presented a method for precomputing multiple scattering events in participating media. These precomputed multiple scattering can be combined with many existing rendering algorithms, reducing computational costs with no visible difference. Our method works with any kind of materials, including refractive materials, high-albedo and low-albedo materials. In future work, we want to study extension to heterogeneous materials.

\section{REFERENCES}

Wenzel Jakob. 2010. Mitsuba Renderer. http://www.mitsuba-renderer.org/. (2010). Wenzel Jakob and Steve Marschner. 2012. Manifold Exploration: A Markov Chain Monte Carlo Technique for Rendering Scenes with Difficult Specular Transport. ACM Trans. Graph. 31, 4, Article 58 (July 2012), 13 pages.

Jaroslav Křivánek. 2014. SmallUPBP. http://www.smallupbp.com/. (2014).

Jaroslav Křivánek, Iliyan Georgiev, Toshiya Hachisuka, Petr Vévoda, Martin Šik, Derek Nowrouzezahrai, and Wojciech Jarosz. 2014. Unifying points, beams, and paths in volumetric light transport simulation. ACM Trans. Graph. (proc. SIGGRAPH) 33, 4, Article 103 (Aug. 2014), 13 pages.

Jonathan T. Moon, Bruce Walter, and Stephen R. Marschner. 2007. Rendering Discrete Random Media Using Precomputed Scattering Solutions. In Rendering Techniques (proc. EGSR). 231-242.

Jan Novák, Derek Nowrouzezahrai, Carsten Dachsbacher, and Wojciech Jarosz. 2012a. Progressive Virtual Beam Lights. Comput. Graph. Forum (Proc. EGSR) 31, 4 (2012), 1407-1413.

Jan Novák, Derek Nowrouzezahrai, Carsten Dachsbacher, and Wojciech Jarosz. 2012b. Virtual Ray Lights for Rendering Scenes with Participating Media. ACM Trans. Graph. (proc. SIGGRAPH) 31, 4, Article 60 (July 2012), 11 pages.

Beibei Wang, Jean-Dominique Gascuel, and Nicolas Holzschuch. 2016. Point-Based Light Transport for Participating Media with Refractive Boundaries. In EGSR (EI\&I). $109-119$

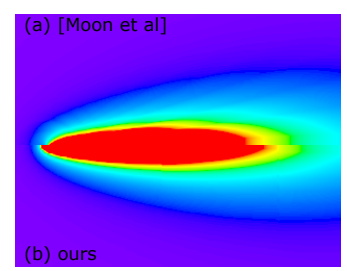

Figure 3: Multiple scattering computed using Moon et al. [2007] (top) and our method (bottom). Moon et al. use concentric spheres, causing angular artifacts, along with low resolution near the axis. 